\title{
Problems in using basal body temperature recordings in an infertility clinic
}

\author{
ELIZABETH A LENTON, GERALD A WESTON, IAN D COOKE
}

British Medical fournal, 1977, 1, 803-805

\begin{abstract}
Summary
Basal body temperature recordings are extensively used to diagnose and treat infertility, but too great an emphasis on the interpretation of these charts might be counterproductive in managing these patients. Several gynaecologists who use temperature charts clinically were asked to score 60 charts taken from a selection of normal and infertile women, and their results were compared with those obtained by a group of non-experts. Since the full hormonal profiles had been obtained for each of the 60 charts the accuracy of the predictions could be assessed. About $80 \%$ of the temperature charts were correctly interpreted by both groups as being either ovulatory or anovulatory, but the day of ovulation was predicted correctly for only about $34 \%$ of the charts. When the charts were examined retrospectively the thermal nadir was found to coincide with the luteinising hormone surge in $43 \%$ of the charts from normal subjects but in only $25 \%$ of those from the infertile patients. Predicting the day of ovulation from the temperature recording, particularly in infertile women, is clearly unjustified.
\end{abstract}

\section{Introduction}

Ovulation and corpus luteum formation are accompanied by a small rise in basal body temperature.' This knowledge has been used both to prevent conception ${ }^{2}$ in normal women and to improve the chances of pregnancy in infertile women. ${ }^{34}$ More recently, however, basal body temperature (BBT) recordings have increasingly been used in infertility clinics, not only to detect anovulation ${ }^{3} 5$ but also to indicate defects in the cycle of the regularly ovulating patient ${ }^{45}$ when it is impractible to perform detailed hormonal profiles. As an intrinsic part of the investigation of infertile patients, BBT charts are used for timing urine collections for hormone assays, postcoital tests, and endometrial biopsies. ${ }^{6}$ When endometrial dating is performed, this too is compared with the age of the corpus luteum as deduced from a BBT chart. ${ }^{8}$

Although the age of the corpus luteum and dating of the endometrium and either the day of the luteinising-hormone (LH) surge or the day of thermal nadir on a BBT recording correlate well in normal women, ${ }^{9}$ we have observed that BBT charts from infertile patients are not so easy to interpret. ${ }^{10} \mathrm{We}$ therefore undertook a study to determine the degree of accuracy and consistency with which a group of observers could assess BBT recordings obtained in these patients.

\section{Subjects and methods}

During other research projects we have accumulated many BBT

University Department of Obstetrics and Gynaecology, Jessop Hospital for Women, Sheffield

ELIZABETH A LENTON, PHD, research biochemist

GERALD A WESTON, medical student

IAN D COOKE, FRCOG, professor of obstetrics and gynaecology recordings of cycles from patients who have also had full daily hormonal profiles constructed, and thus both the day of the LH surge and the type of cycle are known accurately.

Sixty temperature charts (table I) from our records were selected at random, redrawn to exclude the name of the patient but including all relevant clinical details (onset of menses, days and type of treatment where appropriate, etc), and presented to two groups of assessors. The groups were composed of "experts" in interpreting BBT charts (gynaecologists actively looking after infertile patients) and "nonexperts" (general practitioners, junior hospital staff, and technicians, who, although fully conversant with the general features of BBT charts, did not use them clinically).

TABLE I-Types of BBT charts selected at random for use in survey

\begin{tabular}{|c|c|c|c|}
\hline Subjects & & & No of charts \\
\hline 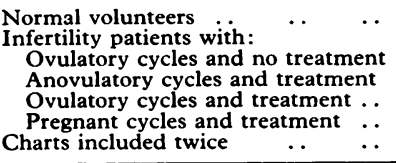 & $\begin{array}{l}\cdots \\
\cdots \\
\cdots \\
\cdots \\
\cdots\end{array}$ & $\begin{array}{ll}\cdots & \cdots \\
\cdots & \cdots \\
\cdots & \cdots \\
\cdots & \cdots \\
\cdots & \cdots\end{array}$ & $\begin{array}{r}8 \\
14 \\
4 \\
24 \\
4 \\
6 \\
\end{array}$ \\
\hline \multicolumn{3}{|r|}{ Total } & 60 \\
\hline
\end{tabular}

The assessors were asked to evaluate the charts and indicate (a) whether the cycle was ovulatory or anovulatory; $(b)$ whether the cycle was normal, defective (from an infertile patient), or one in which conception uccurred; and $(c)$ the day on which they thought ovulation occurred.

The results were scored separately for each of these questions, and the maximum scores obtainable were calculated as follows: $(a)$ one point was awarded for each chart correctly interpreted as ovulatory or anovulatory; $(b)$ one point was awarded for each chart correctly diagnosed as being a normal, defective, or showing conception; and (c) one point was awarded if the answer coincided with or was the day after the LH surge. Our hormone assays indicated only the day of the LH surge, and ovulation is thought to occur within 12-24 hours of the LH surge. ${ }^{11} 12$

To test consistency six of the charts were given twice to each assessor, and one mark was awarded for each pair of charts that had been assessed identically regardless of whether this assessment was correct.

\section{Results}

The mean scores obtained from the two groups (experts and nonexperts) were expressed as percentages of the total marks obtainable (table II). While the experts obtained slightly better results than the non-experts, neither group did particularly well. About $80 \%$ of the charts were correctly assessed as either ovulatory or anovulatory, but only about half of them were correctly interpreted as coming from either a normal or a defective cycle or a pregnant woman.

TABLE II-Mean ( $\pm S D$ ) scores (expressed as percentages) obtained by the experts and non-experts in assessing temperature charts

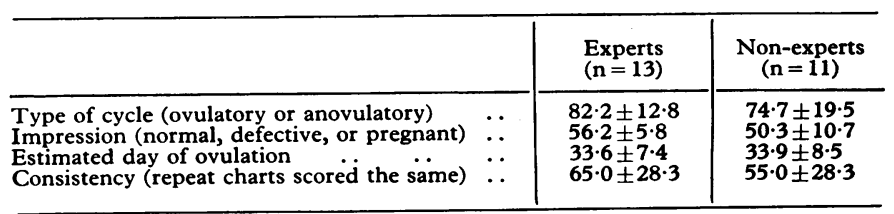


The mean scores for the day of ovulation showed that even when a time span of two days was permitted only a few of the cycles were correctly assessed. The small standard deviation reflected the fact that most of the assessors gave similar responses.

There were relatively few charts from normal controls, but this reflects conditions in most infertility clinics. As the information deduced from temperature charts is used most extensively in infertility clinics, it seemed worth while to attempt to explain the reason for the low rate of success in predicting the day of ovulation. Accordingly all the charts were carefully re-examined and the three main types of cycle-"normal" from a control subject; "defective" from an ovulatory but infertile patient; and "treated" from an infertile patient being treated with clomiphene-were analysed separately.

The mean length of the cycle was three to four days longer in the treated group (as a result of longer follicular and luteal phases; table III) than in either of the other two types of cycle. The day of the $\mathrm{LH}$ surge was designated day 0 , so the sum of the follicular and

TABLE III-Mean $( \pm S D)$ lengths of follicular and luteal phases and of cycle (days) for normal, defective, and treated cycles

\begin{tabular}{|c|c|c|c|}
\hline & $\begin{array}{l}\text { Normal } \\
(n=8)\end{array}$ & $\begin{array}{l}\text { Defective } \\
(n=14)\end{array}$ & $\begin{array}{l}\text { Treated } \\
(n=24)\end{array}$ \\
\hline $\begin{array}{l}\text { Length of follicular phase } \\
\text { Length of luteal phase } \\
\text { Cycle length }\end{array}$ & $\begin{array}{l}12 \cdot 5 \pm 2 \cdot 5 \\
13 \cdot 6 \pm 1 \cdot 9 \\
27 \cdot 1 \pm 3 \cdot 6\end{array}$ & $\begin{array}{l}12 \cdot 9 \pm 2 \cdot 1 \\
13 \cdot 6 \pm 2 \cdot 7 \\
27 \cdot 5 \pm 2 \cdot 1\end{array}$ & $\begin{array}{l}15 \cdot 5 \pm 2 \cdot 9 \\
15 \cdot 0 \pm 1 \cdot 9 \\
31 \cdot 5 \pm 3 \cdot 0\end{array}$ \\
\hline
\end{tabular}

TABLE IV-Distribution (\%) of day of the thermal nadir with respect to day of LH surge for three main types of cycle

\begin{tabular}{c|c|c|c}
\hline $\begin{array}{c}\text { Day of thermal nadir relative } \\
\text { to day of } \mathrm{LH} \text { surge (day 0) }\end{array}$ & $\begin{array}{c}\text { Normal } \\
(\mathrm{n}=7)\end{array}$ & $\begin{array}{c}\text { Defective } \\
(\mathbf{n}=12)\end{array}$ & $\begin{array}{c}\text { Treated } \\
(\mathbf{n}=\mathbf{2 8})\end{array}$ \\
\cline { 1 - 3 } Day -1 & 29 & 17 & 32 \\
Day 0 & 43 & 25 & 25 \\
Day+1 & 14 & 26 & 22 \\
\hline
\end{tabular}
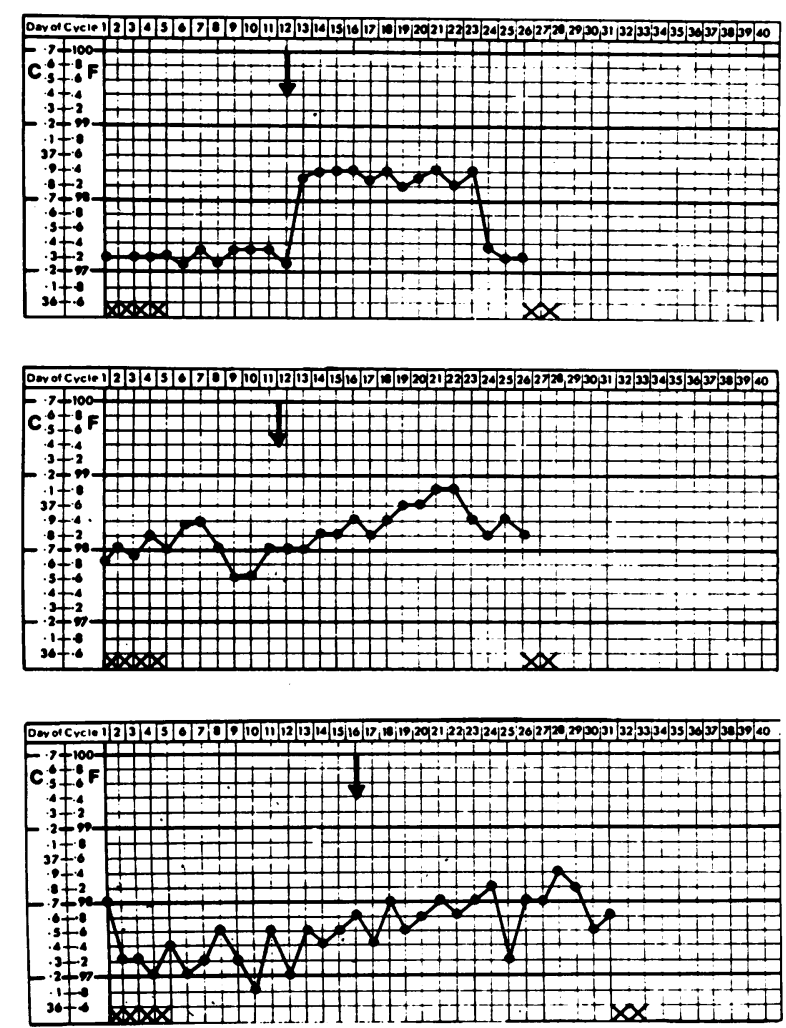

FIG 1-Three examples of temperature charts. Top chart shows normal characteristics, and lower two are characteristic of those often seen in infertility clinics. All these cycles were ovulatory. Day of LH surge is arrowed.
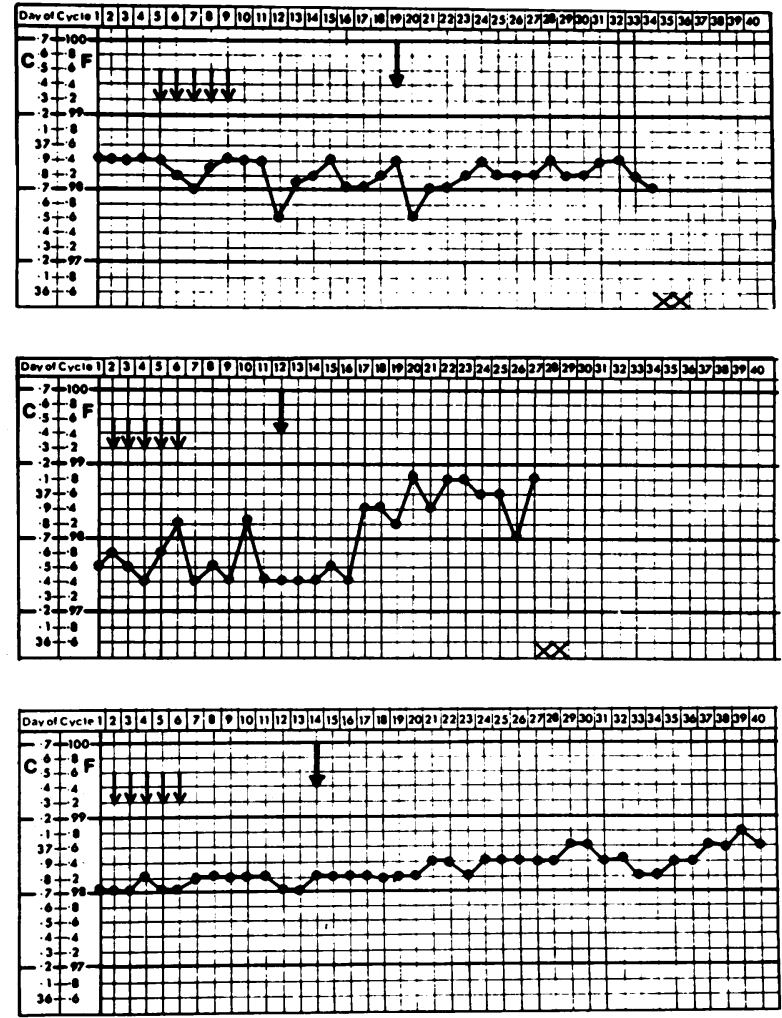

FIG 2-Three examples of ambiguous or misleading temperature charts in patients treated with clomiphene (small arrows). All cycles were ovulatory, and day of the LH surge is marked with large arrow. Bottom chart is of cycle in which conception occurred.

luteal phases was equivalent to the cycle length less one day. Thus there was an appreciable error in retrospectively pinpointing the day of ovulation by counting backwards from the first day of the next menstrual period.

In any case, decisions about treatment or the timing of investigations often have to be made before the length of the cycle is known, although inferences are drawn from previous cycles.

The features of a BBT chart associated with ovulation are the slight drop in temperature (thermal nadir) ${ }^{1}$ followed by a sharp rise of usually about 0.12 to $0.3^{\circ} \mathrm{C}\left(0.4\right.$ to $\left.1.0^{\circ} \mathrm{F}\right)$. The temperature is then maintained at this higher level until the end of the luteal phase. When the day of the thermal nadir (determined retrospectively by taking the most appropriate dip that fitted with the LH surge) was correlated with the day of the LH surge a significant correlation $(r=0.86)$ was obtained. ${ }^{11}$ Careful examination of the regression line showed that the scatter of points in the defective cycles was greater than that in either the normal or the treatment cycles. This information is summarised in table IV, which shows clearly that although the thermal nadir occurred 0-24 hours before the LH surge in $72 \%$ of normal cycles, it did so in only $42 \%$ of defective cycles. Thus any prediction of the time of ovulation will be wrong twice as often in cycles from infertility patients as in cycles from normal subjects.

Examples of temperature charts that were particularly difficult to interpret or were frankly misleading are shown in figs 1 and 2 .

Marks given for the degree of consistency shown by each assessor indicated that most were in fact guessing the day of ovulation, even though they thought that they were maintaining a high degree of consistency. Only four $(16.7 \%)$ participants marked each pair of the six repeated cycles identically and a further five $(20.8 \%)$ marked five pairs the same.

\section{Discussion}

Although most of the temperature charts in this series were correctly assessed for the presence or absence of ovulation, few had the day of ovulation predicted correctly..$^{13} 14$ One of the reasons for this appeared to be that the characteristic features of a normal chart-that is, a thermal nadir followed by an abrupt 
rise in temperature-were absent in many of the cycles, even though these were ovulatory by hormonal analysis (fig 1), and when these features were present they were not consistently related to the timing of the LH surge (table IV).

Most of the charts included in the series were from infertile patients and were obtained either before or during treatment. These were included in an attempt to simulate conditions in an infertility clinic. Since the classical changes seen in a BBT are only indirectly related to ovulation-namely, $\mathrm{LH}$ surge, rupture of the follicle, formation of corpus luteum, synthesis of progesterone, ${ }^{12}$ rise in $\mathrm{BBT}^{4}$ - not surprisingly an infertile cycle (despite being ovulatory) may well give rise to an atypical BBT chart. This observation is not new. ${ }^{315}$ Indeed, abnormalities in BBT charts from infertile patients have often been described, ${ }^{916-18}$ though it is still commonly assumed that the day of ovulation can be predicted fairly accurately. As a result the condition of the cervical mucus ${ }^{1419}$ or the results of isolated hormone assays may be wrongly assessed. Moreover, couples are often advised on the best time to have intercourse or attend hospital for artificial insemination on the basis of BBT recordings. ${ }^{3}$

Although we have emphasised the errors in interpreting charts from infertile patients, an appreciable number of the normal cycles were also scored wrongly. ${ }^{14}$ Even under optimum conditions-that is, retrospective assessment of the chart with prior knowledge of the day of the $\mathrm{LH}$ surge-only $72 \%$ of the normal cycles showed the conventional features correlating with hormonal events (table IV).

All charts were marked within 20 minutes, so an even lower degree of consistency would be achieved by anyone reviewing the charts produced by a patient at monthly intervals.

Unfortunately the cost and technical difficulties of obtaining hormonal profiles prohibits their routine use in an infertility clinic, so the clinician does not have regular feedback on the accuracy of his interpretations and therefore no chance of improving his future assessments.

Despite these drawbacks the use of BBT recordings in an infertility clinic is probably still justified as a means of providing a concise visual record of menstrual dates, times of hormone assays, results of biopsies, and duration and dose of any treatment, so long as the temptation to draw detailed conclusions about the progress of hormonal events is avoided. ${ }^{13} 20$

The willing co-operation of all participants, both expert and nonexpert, is gratefully acknowledged. We should also like to thank Mrs Janet Southall for scoring all the result sheets.

\section{References}

${ }^{1}$ Halbrecht, I, Lancet, 1945, $2,668$.

2 Marshall, J, British Medical fournal, 1963, 1, 102.

${ }^{3}$ Benjamin, F, fournal of Obstetrics and Gynaecology of the British Commonwealth, 1960, 67, 177

4 Akin, A, and Elstein, M, International fournal of Fertility, 1975, 20, 122.

5 Johansson, E D B, Larsson-Cohn, U, and Gemzell, C A, American fournal of Obstetrics and Gynecology, 1972, 112, 933.

${ }^{6}$ Murthy, Y S, Arronet, G H, and Parekh, M C, Obstetrics and Gynecology, 1970, 36, 758.

${ }^{7}$ Noyes, R W, Hertig, A T, and Rock, J, Fertility and Sterility, 1950, 1, 3.

8 Cooke, I D, and Lambadarios, C, in Clinics in Obstetrics and Gynaecology, ed I D Cooke, vol 1, No 2, p 369. Philadelphia, Saunders, 1974.

${ }^{9}$ Lundy, L E, et al, Obstetrics and Gynecology, 1974, 44, 14.

10 Adams, M, and Cooke, I D, in Clinics in Obstetrics and Gynaecology, ed I D Cooke, vol 1, No 2, p 285. Philadelphia, Saunders, 1974.

11 Croxatto, H B, et al, Fertility and Sterility, 1972, 23, 447.

12 Yussman, $\mathrm{M} \mathrm{A}$, and Taymor, M L, fournal of Clinical Endocrinology and Metabolism, 1970, 30, 396.

${ }^{13}$ Buxton, C L, and Engle, E T, American fournal of Obstetrics and Gynecology, 1950, 60, 539.

14 Polishuk, W E, and Diamant, Y Z, Fertility and Sterility, 1973, 24, 245.

15 Arratta, W S M, and Iffey, L, Obstetric and Gynaecological Survey, 1971, 26, 675 .

16 Jones, G E S, fournal of the American Medical Association, 1949, 141, 112

17 Murray, M, and Osmond-Clarke, F, fournal of Obstetrics and Gynaecology of the British Commonwealth, 1971, 78, 1108.

18 Strott, C A, et al, fournal of Clinical Endocrinology and Metabolism, $1970,30,246$.

10 Elstein, M, in Clinics in Obstetrics and Gynaecology, ed I D Cooke, vol 1 , No 2, p 345. Philadelphia, Saunders, 1974.

20 Siegler, S L, and Siegler, A M, Fertility and Sterility, 1951, 2, 287.

(Accepted 18 fanuary 1977) other studies. In at least 256 incidents $(27 \%)$ there was a history of repeated overdose in the previous 12 months. Comparison with other studies showed a steady decline in the use of barbiturates in deliberate selfpoisoning but an increase in the use of non-barbiturate hypnotics, minor tranquillisers, and antidepressants.

\section{Introduction}

The problem of attempted suicide has been studied intensively in Britain during the last 20 years in rural ${ }^{1}$ and urban ${ }^{2-4}$ areas and many studies suggest that its incidence is increasing. ${ }^{24-6}$ This appears to be accounted for by a massive increase in the number of cases of deliberate self-poisoning and even the most recent studies show that the peak of this "epidemic" has not yet been reached. There is also evidence that the incidence of accidental overdose and of drug dependency has been increasing too, ${ }^{78}$ and the present study concentrates on cases of deliberate 